\title{
Exploring Communication Processes in Referral Pathways for Chronic Disease Management: Malaysian Public Primary Health care Experiences
}

\author{
Zalilah Abdullah ${ }^{1}$, Low Lee Lan ${ }^{1}$, Iqbal Ab Rahim ${ }^{1}$, Syafinas Azam ${ }^{1}$, \\ Mohammad Zabri Johari ${ }^{2} \&$ Nazrila Hairizan Nasir ${ }^{3}$ \\ ${ }^{1}$ Institute for Health Systems Research, National Institutes of Health, Ministry of Health Malaysia, Selangor, \\ Malaysia \\ ${ }^{2}$ Institute for Health Behavioural Research, National Institutes of Health, Ministry of Health Malaysia, Selangor, \\ Malaysia \\ ${ }^{3}$ Family Health Development Division, Ministry of Health Malaysia, Putrajaya, Malaysia \\ Correspondence: Zalilah Abdullah, Institute for Health Systems Research, National Institutes of Health, Ministry \\ of Health Malaysia, Setia Alam, 40170, Selangor, Malaysia. Tel: 603-33627500 ext. 8505. Fax: 603-33-627-501. \\ E-mail: drzalilah@gmail.com/zalilah.a@moh.gov.my
}

Received: August 4, 2020 Accepted: September 23, 2020 Online Published: November 11, 2020

doi:10.5539/gjhs.v12n13p115 URL: https://doi.org/10.5539/gjhs.v12n13p115

\begin{abstract}
Background: Referrals are a two-way communication between healthcare facilities to enable information transfer for the continuity of patient care. The Enhanced Primary Healthcare (EnPHC) initiative, a complex intervention package to improve non-communicable disease (NCD) management, introduced improvements to the NCD's referral mechanism from primary healthcare clinics to the hospital. This study explores the communication process between the Malaysian public primary healthcare and hospital for chronic care management.
\end{abstract}

Method: A qualitative exploratory study using purposive sampling was done in all twenty EnPHC intervention clinics. In-depth interviews and focus group discussions were carried out among all healthcare providers working in EnPHC clinics. The 47 interview sessions were audio-recorded, transcribed verbatim, and analyzed thematically.

Results: A total of 97 healthcare providers participated. Three main themes of the communication process between the primary health care and hospital during the implementation of EnPHC intervention emerged from the analysis. These are; (1) structured information relay, (2) no show appointment tracking via various communication devices has strengthened the mechanism to monitor the referred patient appointment scheduling and their adherence to the appointment, and (3) inter-facility networking facilitated the implementation of EnPHC's referral mechanism.

Conclusion: The EnPHC referral mechanism created a platform for PHC clinics and hospitals to communicate and build rapport to help ensure care continuity for NCD patients. The traditional method of communication between healthcare facilities should change and instead start using the newest or most current, advanced technology.

Keywords: Communication, primary healthcare, referral system

\section{Introduction}

A smooth referral process between primary healthcare providers in clinics and specialists in hospitals is fundamental for patient care management. It is a two-way communication where both doctors are responsible to communicate clearly with the patient on the need(s) and reason(s) for the referral (Jarallah, 1998) and deliver feedback on his/her evaluation of the patient's condition. The receiving specialists need to have sufficient information regarding a patient's health status, and for further management, to derive a working treatment plan. A previous study on the coordination of care between primary care doctors and hospitals showed a $43 \%$ dissatisfaction with the quality of information during referrals, and less than $50 \%$ of primary care doctors received 
information or discharge summaries regarding the patients they were referred (Bodenheimer, 2008; Gandhi et al., 2000). This shows that a lack of effective communication between referring physicians can result in poor and inappropriate information transfer that can impact patient management and cause dissatisfaction amongst health care providers (Ang et al., 2014; Wong et al., 2019).

Challenges regarding referrals include the burden on patients to obtain referral appointments, patients' demand for referrals, and patients' compliance to referral appointments (Ministry of Health Malaysia, 2017; Senitan, Alhaiti, \& Gillespie, 2019; WHO, n.d.). Patients might not show up for their allocated referred time due to reasons such as long waiting times at health care facilities, unsuitable appointment times or the patient themselves perceive their health issues as resolved(Forrest, Shadmi, Nutting, \& Starfield, 2007; van Dijk et al., 2016). Malaysia's National Referral System consists of clear guidelines for referral processes with the ultimate goal of providing greater equity, accessibility, and better resource utilisation (Merican \& bin Yon, 2002). However, currently, $50.2 \%$ of the primary healthcare doctors interviewed explained that they seldom or never received any feedback from the hospital specialist regarding treatments or diagnoses of the patients they were referred to (Ministry of Health Malaysia, 2017). Evaluation studies on Malaysia's referral system are lacking and would be important to fill the gap between what is known about the referral system, regardless of the positive effects or challenges, and what is unknown.

The Malaysian Ministry of Health has embarked on the Enhanced Primary Healthcare (EnPHC) initiative, a complex intervention package that introduces improvements to the non-communicable disease (NCD) care management in the primary healthcare setting (Ministry of Health Malaysia, 2017). The pilot implementation was carried out in two States in Malaysia; Selangor and Johor. One of its many interventions was to improve the NCD patient referral process between primary healthcare clinics and hospitals. Three implementation strategies were developed for this intervention; (i) the introduction of the NCD Care Form as the standardised referral communication tool to ensure care continuity between clinics and hospitals, (ii) establishment of liaison officers (LO) in clinics and hospitals for the management of patient referral appointments, and (iii) establishment of inter-facility information sharing platform for discussion, monitoring, and strengthening inter-facility communication (Ministry of Health Malaysia, 2017). Diagram 1 shows the referral workflow processes.

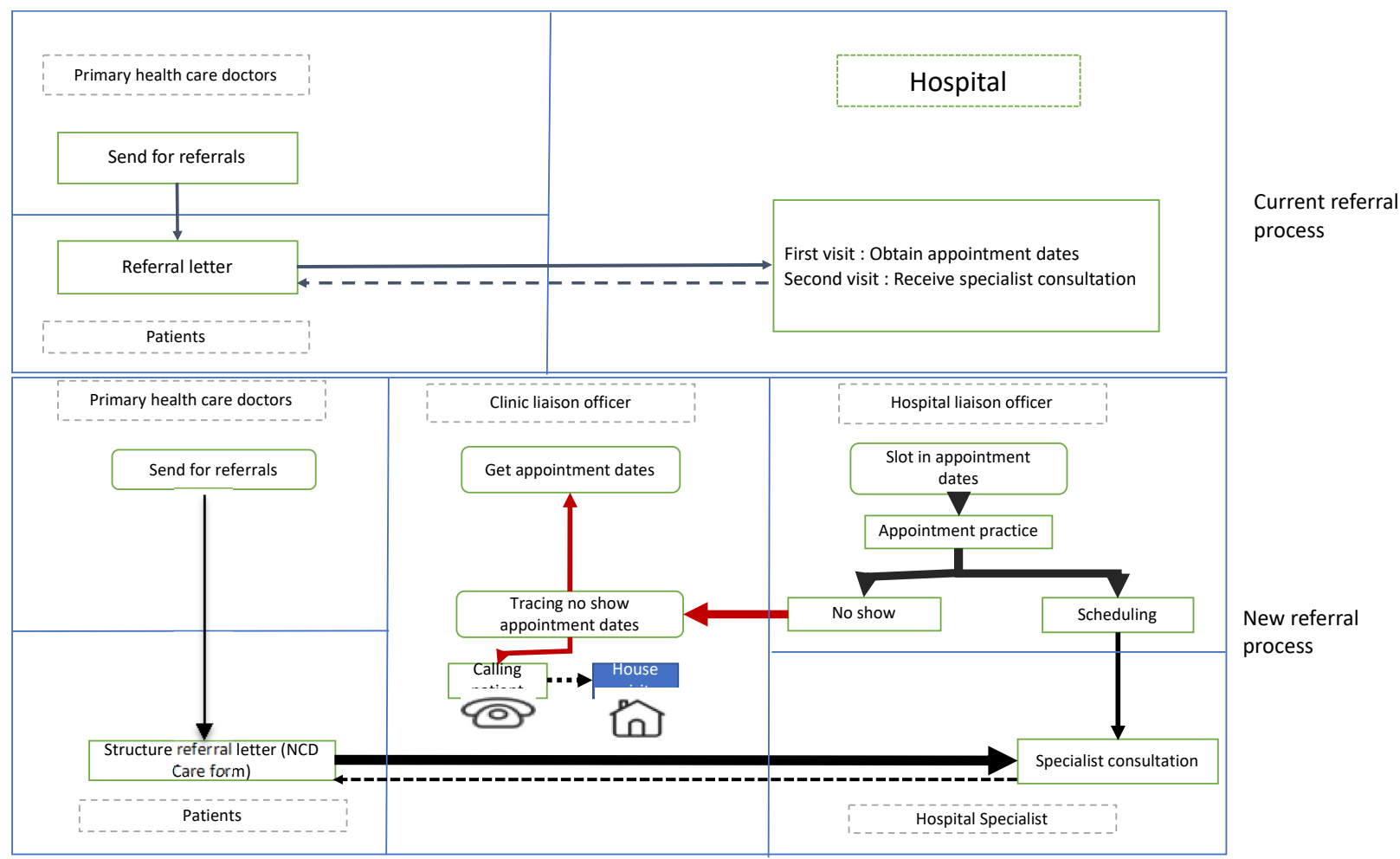

Diagram 1. Current and new referral workflow process

The NCD Care Form is a tool with standardised formatting for data input to minimise inconsistencies in the comprehensiveness and specificity of information. Information such as patient background, screening results, diagnosis, complications, vital signs, medication intake, medical history, referral, and management plans are all 
contained within the tool. The referral section consists of the referral appointment date, reason for referral, and feedback summary to the referring party. LOs are appointed among healthcare providers (HCP) in both clinics and hospitals. Part of the responsibility was to receive and organise inter-facility referrals, monitor the referred patient's adherence to the appointment, and to collate and channel feedback regarding the appointments. Indirectly, this strategy eliminated the need for the patient to obtain their referral appointments. A communication platform between the primary healthcare clinics (PHC) and hospitals was also institutionalised by quarterly meetings for structured information sharing, monitoring, and strengthening of inter-facility communication (Ministry of Health Malaysia, 2017). These implementation strategies for EnPHC's referral system were assessed as part of the process evaluation study. Hence, this paper aims to explore the communication processes in referral pathways for Chronic Disease Management.

\section{Method}

\subsection{Study Design and Sampling}

A qualitative exploratory study using in-depth interview (IDI) and focus group discussion (FGD) among HCPs in the public PHC clinic (hereafter referred to as PHC clinic), the EnPHC intervention design team, and State coordinators. Purposive sampling was used for this study. A sampling of HCPs as participants of the focus group discussion was identified as the clinic's staff, where the criteria were for the staff to be involved in the implementation of the intervention. Those who fulfiled the HCPs' criteria during our data collection were approached to participate in FGD and IDI, and each person approached were allowed to refuse participation in the interviews. The sample size was determined by data saturation where no new information emerged after multiple probing questions were asked during interviews.

\subsection{Ethical Approval}

Ethical approval for the EnPHC study was obtained from the Medical Research Ethics Committee (MREC) Malaysia (Research registration number: NMRR-17-295-34771). Informed written consent was collected from all participants before interviews.

\subsection{Data Collection and Data Analysis}

Data collection was conducted in both States of Selangor and Johor, in all PHC clinics participating in the EnPHC initiative, at two different periods during the implementation. This was to ensure that participating clinics implemented the intervention and to obtain a confluence triangulation of data to support the evaluation's findings. Round one of data collection was conducted over a three-month period (months three to five) during implementation, and follow up data collection for round two was conducted over another four-month period (months nine to twelve) during implementation. The interview sessions with HCPs were carried out in PHC clinics to allow for participants' comfort and convenience, and most interviews were done during clinic breaks to ensure no interruption of clinic flow. State coordinators and EnPHC intervention design team members' interview sessions were conducted in their respective workplaces. Interviews with State coordinators and the EnPHC intervention design team were scheduled to bring clarity to the gaps in information made by the clinics. All interviews were conducted in a quiet, secure, and comfortable room. Interview sessions were conducted face-to-face by research team members trained in the qualitative method and were not close acquaintances of any of the participants, to avoid potential response bias.

All HCPs (doctors, pharmacists, medical assistants, nurses, laboratory staff, clinic attendants, clerks) considered for IDI are those in managerial positions: Liaison officers (LO) appointed to oversee the implementation process in each clinic and liaise with the policy maker-in-charge of EnPHC, and medical assistant or registered nurse appointed as Care coordinator (CC) \& State Coordinator. The LO \& CC are the leaders of the intervention tasks at the clinic. We separated them from the HCPs of the FGD to facilitate the discussion and avoid response bias on the perception of other HCPs of the FGD and vice versa. The State Coordinators were interviewed separately as they are stationed at the State Health office. FGD among participants from the intervention design team were purposively selected from person who is most experienced in various disciplines (primary health care setting and hospital setting) and involved in the overall designing of intervention component. All HCPs working in PHC clinics were interviewed using a semi-structured interview guide aimed at exploring the experiences and issues encountered with the EnPHC's referral system. The interview guide consisted of questions on the actual referral system's implementation between PHC clinics and hospitals, feasibility (challenges encountered), adaptations to local contexts, and perceived early benefits of the intervention. The topics covered by the interview guide for state coordinators and the EnPHC intervention design team consisted of the intervention's design and characteristics, execution plans at different levels (headquarters and states), and the engagement mechanism for the development 
of the intervention. Interviews were conducted either in Malay, English, or in mixed languages, based on the participants' level of understanding; the researchers were fluent in either language. Participants were also asked a series of probing questions related to monitoring activities, adaptation in local contexts, and management of issues encountered by intervention clinics.

Participant information sheet and informed consent were given to participants before the interviews, and all approached participants agreed to be interviewed. A total of 10 FGD sessions and 38 IDI sessions were conducted, each lasting 30 to 120 minutes. Round one recorded 20 IDI sessions with HCPs, one FGD with the intervention's design team, and two IDI sessions with the state coordinators, while round two recorded nine FGD sessions and 14 IDI sessions with a total of 69 HCPs. All interviews were audio-recorded and later transcribed verbatim. During the interview, the facilitator will often summarise feedback given to decrease the incidence of incorrect data and the incorrect interpretation of data. Field notes were also taken by the note-taker during the interviews. Confidentiality was ensured by removing participants' identifiers from transcripts. Each transcript was cross-checked by the research team through audio listening and field notes.

Transcripts were read repeatedly to obtain a full understanding before each transcript was open-coded independently by researchers from a multidisciplinary background (physician, behavioural scientist, health sciences). Thematic analysed were performed. The coding process was done independently in pairs and the same transcript was read by two persons in the research team, each who coded it separately. After the level one coding, all similar coding were grouped to form a category, then, followed by consensual validation among the researchers before, codes and verbatim quotes were regrouped into major themes (examples of a coding process as in table 1). The first-order and second-order coding approach was applied during analysis (Gioia, Corley, \& Hamilton, 2012). Any coding discrepancies were resolved with a series of discussions among the research team to reach a consensus.

Table 1. Example of coding process

\begin{tabular}{|c|c|c|c|}
\hline Verbatim & Level 1 & $\begin{array}{l}\text { Level } 2 \\
\text { (category) }\end{array}$ & Theme \\
\hline $\begin{array}{l}\text { "It is easy for the EnPHC patient because they use the same } \\
\text { NCD care form so we do not need to fill in other form. NCD care } \\
\text { form has all information needed in it" (Doctor, } 34 \text { years old). }\end{array}$ & $\begin{array}{l}\text { NCD care form } \\
\text { provides information } \\
\text { to the receiving } \\
\text { specialist. }\end{array}$ & \multirow{2}{*}{$\begin{array}{l}\text { New tool for } \\
\text { information } \\
\text { transfer }\end{array}$} & \multirow{3}{*}{$\begin{array}{l}\text { Structured } \\
\text { information } \\
\text { relay }\end{array}$} \\
\hline $\begin{array}{l}\text { "All the medications are written on the NCD care form, usually } \\
\text { a patient referred to hospitals they rarely bring their TCA } \\
\text { (patient appointment book) by using NCD care form they } \\
\text { (Specialist at Hospital) can see what are the medication patient' } \\
\text { taking". (Pharmacist, } 29 \text { years old.) }\end{array}$ & $\begin{array}{l}\text { Medications are } \\
\text { written on the NCD } \\
\text { care form }\end{array}$ & & \\
\hline $\begin{array}{l}\text { "Space in NCD care form too small to include comment from } \\
\text { hospital, it is not enough. However, they (hospital) attached } \\
\text { extra paper (for feedback)" (Doctor, } 48 \text { years old). }\end{array}$ & $\begin{array}{l}\text { Limited space in the } \\
\text { NCD care form }\end{array}$ & $\begin{array}{l}\text { Design of NCD } \\
\text { care form }\end{array}$ & \\
\hline
\end{tabular}

\section{Results}

This qualitative exploratory study involved participants from various levels and categories of HCPs. Table 2 presents the participants' demographics. There were 97 participants; 91 HCPs from various categories at intervention clinics, two state coordinators, and four members of the intervention design team. Three themes emerged from the analysis; 'structured information relay', 'no-show appointment tracking', and 'inter-facility networking'. 
Table 2. Demographic of participants in EnPHC's implementation study

\begin{tabular}{|c|c|c|}
\hline \multirow{2}{*}{ Participants } & \multicolumn{2}{|l|}{ No of participants } \\
\hline & In-depth Interview & Focus group discussion \\
\hline $\begin{array}{l}\text { State Coordinator } \\
\qquad \quad \text { Medical Doctor }\end{array}$ & 2 & \\
\hline $\begin{array}{l}\text { Intervention Design team } \\
\bullet \quad \text { Medical Doctor }\end{array}$ & & 4 \\
\hline \multicolumn{3}{|l|}{ Health Care Provider } \\
\hline - Medical Doctor & 19 & 14 \\
\hline - $\quad$ Registered Nurse & 9 & 15 \\
\hline - Medical Assistant & 7 & 8 \\
\hline - $\quad$ Pharmacist & & 10 \\
\hline - Others & 1 & 8 \\
\hline Total & 38 & 59 \\
\hline Grand total & 97 & \\
\hline
\end{tabular}

\subsection{Structured Information Relay}

New tool for information transfer. One of the three implementation strategies for improvements in the NCD care referral system was the introduction of the NCD care form as a standardised tool. HCPs conveyed their approval of the tool's usage as it facilitated the patient care management process. The tool was also found to be easy to use and informative as it listed the reasons for visits, medications, and laboratory blood investigations in a structured manner. These findings were supported by the following selected quotes.

"All the medications are written on the NCD care form, usually a patient referred to hospitals they rarely bring their TCA (patient appointment book) by using NCD care form they (Specialist at Hospital) can see what is the medication patient's taking". (Pharmacist, 29 years old.)

Design of NCD care form. Regarding the usability of the tools, views were shared for improvements to the tool's design, specifically the section to communicate the patient's management plan, as shared by a participant, "Space in NCD care form too small to include comment from the hospital, it is not enough. However, they (hospital) attached extra paper (for feedback)" (Doctor, 48 years old).

Feedback from hospitals regarding referrals from the hospital (also referred to as counter-referral) was found to still be lacking at the point of interviews. The concern regarding the inadequacy of the feedback information loop was voiced out by the staff, "We just want to know what happens, because we don't know whether the diagnosis was right or not. At least when they tell us this was done, then okay. Otherwise, we just assume." (Doctor, 31 years old). As shown in Diagram 1, the evaluation also found the intervention design did not take into account this particular mechanism; feedback from the hospitals to the physicians in the PHC clinic was handed directly to the patient. If the patient returned to the clinic to continue treatment, and if the patient remembered to bring the feedback along, the counter-referral information will be received at a delayed-pace. This was one of the identified areas for improvement concerning the tool's design and usability.

Awareness among the specialists in the hospital regarding the usage of the NCD care form is equally important for the intervention to be successfully implemented. This finding was voiced out by the intervention team during their assessment visit to the hospital: "the hospital director acknowledged Specialist in the hospital did not know. The Hospital director took the responsibility to create awareness to the Specialists. Because if the specialists do not aware of the form (NCD care form), they will be reluctant to use the form. So that's why we asked the hospital director to create awareness program for all these specialists." (Intervention team: Doctor, 55 years old).

\subsection{No-Show Appointment Tracking}

Through the EnPHC initiative, patients no longer have to travel to get a hospital appointment date whenever they are referred from the PHC clinic. This is because the appointments are managed directly by the LOs in both facilities. The LO in the hospital can either be the matron- or nurse-in-charge or the head clerk of the specialist 
clinic while the LO in the PHC is generally the care coordinator, who would either be a medical assistant or a nurse. The introduction of the LO roles in both clinics and hospitals were found to have a positive effect in monitoring the patients' compliance with the referral appointments. As both LOs are in constant communication for referrals' appointment scheduling, they also provide feedback to one another on whether the referred patient turned up for the appointment. This communication channel established a no-show appointment tracking system for the referred patient, which can minimise incidences of defaulted referral appointments. This is an important component in ensuring care continuity. These findings were supported by the following selected verbatim quote.

"So far, to the Hospital C, care coordinator will manage all appointments. That means care coordinator will call LO specialist clinic in Hospital C for appointment dates. So if there is appointment's defaulter, LO hospital will inform us. "(Nurse, 30 years old)

Additionally, some PHC clinics established a referral registry to facilitate the monitoring process. As informed by a participant, "We have that registry...from the registry we able know when are the appointment dates. Here, how we do the referrals is that (name of identified personnel) will call the hospital, will check registry daily...he will call the eye clinic whether the patient show up for appointment or not later this evening." (Doctor, 48 years old)

\subsection{Inter-Facility Networking}

Inter-facility communication was recognised as a longstanding issue before EnPHC. However, since improvements to the NCD care referral system required participation from both PHC clinics and hospitals, two-way communication needed to be established to improve inter-facility coordination. Early benefits from the EnPHC's implementation were seen and recognised by the state health coordinators, who said, "Referral system becomes faster, patients do not need to go to the hospital anymore [for appointment date] as the appointment already been arranged. The system-interlink between hospital and primary healthcare clinic looks good" (State Coordinator, 41 years old). Additionally, some hospitals took the initiative to allocate specific appointment slots to receive referrals from PHC clinics. As shared by a participant, "MOPD [Medical Out-patient Department] in hospital $E$ has already allocated two patients every Wednesday. We do not need to call the hospital as we know we can send two patients every Wednesday." (Doctor, 35 years old). This theme can be further expanded into three sub-themes - technology-aided communication, leadership role and discussion platform.

\subsubsection{Technology-Aided Communication}

During implementation, modifications and adaptations were made to improve communication between facilities. In actuality, a mobile phone communication application was used as the communication medium of choice. The utilisation of a mobile phone application for communication between the LOs in PHC Clinics and hospitals to inform one another has thus facilitated fast and easy information sharing for their monitoring activities, as shared by a participant, "I asked LO chief of hospital B, to create a social networking group as what hospital $C$ and $D$ already have done. It is very useful as their LO chief can use the mobile app to monitor the appointment management" (Medical Assistant, 35 years old). The application is also used for appointment management purposes, either to secure hospital appointment dates for referrals from PHC clinics or to alert one another regarding any changes with appointments. This is in line with this selected quote: "Currently, we had referred (to Hospital) three to four patients, the response was ok, because we also have WhatsApp group with LO at the hospital, so every information example like changes of TCA (appointment dates), she will inform us". (Nurse, 34 years old)

\subsubsection{Leadership Role}

HCPs perceived that organisational leadership was important to facilitate the implementation. They seemed to have an ingrained belief that only a central directive can smoothen the implementation's progress, as informed by a participant, "Referral system to the hospital, when involving two other organizations, I think it might be a problem too, because involve another directive from their (hospital) Director, if there is no direction from the superiors (top management), I do not think the system will move, so if there are any written instructions, it will be the strong evidence that (the system) is needed." (Medical Assistant, 37 years old). However, there are also concerns among the coordinators at the headquarters and state-levels that HCPs need to be more assertive in the implementation and be less dependent on directives. This is because all training and guideline documents for the implementation have been provided. This is in line with a selected quote from a state coordinator: "referrals and counter referrals... We include hospitals in State A as well as outside of State A ... we have one for coordination meeting and also briefing ....we brief everyone about Enhanced Primary Healthcare intervention before we start with training." (State coordinator, 49 years old). By considering both statements, the incongruence of feedback showed that better improvements in organizational communication and information sharing from the top 
management or authority figures are needed.

\subsubsection{Discussion Platform}

As networking has been an issue, the strategy to establish a scheduled periodic discussion platform between clinics and hospitals was well received by the implementers. The discussion platform opened up opportunities for HCPs in both facilities to voice out issues on implementation, as shared by a participant, "...usually a meeting with this hospital, the Medical Officer in charge and District officer will attend this meeting. Issues related to this will be voice out by them in the meeting." (Medical Assistant, 37 years old). This platform enabled them to confer and work out solutions to overcome complications together.

\section{Discussion}

Coordination on referral management between the PHC clinics and hospitals is crucial to ensure care continuity, most especially for NCD cases, due to the chronic nature of the illness that requires long term treatment. A structured referral letter is one of ten factors that contribute towards an effective referral system (Senitan, Alhaiti, \& Lenon, 2018). The information within the letter should convey medical information based on scientific knowledge and administrative information to enable the referral process functions effectively and efficiently while ensuring the accuracy of the information, thus also protecting the patient's health (Ramanayake, 2013; Senitan et al., 2019). A systematic review looking at outpatient referrals shows that the use of structured referrals led to the improved pre-referral investigation of patients before referral. In some ways, a structured referral form acts as a checklist, prompting the primary healthcare physician to include important elements of information before referral (Akbari et al., 2008; Greenwood-Lee, Jewett, Woodhouse, \& Marshall, 2018; Wåhlberg, Valle, Malm, \& Broderstad, 2015). Improvements in the volume of documentary communication across the referral pathway may influence patient care in chronic disease management (Jiwa, Walters, \& Mathers, 2004).

Referrals must go both ways, highlighting the need for a similar standard counter-referral or feedback report (Abdelwahid, Al-Shahrani, Elsaba, \& Elmorshedi, 2010). A lack of effective communication between two referring physicians can result in poor and inappropriate information transfer, which could contribute to patient management issues such as the continuation of incorrect treatment, delays in care, and dissatisfaction among healthcare providers (O'Malley \& Reschovsky, 2011; Shannon, 2012; Woolf, Kuzel, Dovey, \& Phillips, 2004). Of the many reasons to introduce the NCD care form, one important reason was for it to be a common tool for hospitals to provide feedback and guidance on further management of care to the referring physician at PHC clinics (Ministry of Health Malaysia, 2017).

Care management support from other healthcare providers regarding the monitoring of appointments and no show tracking between hospitals and primary healthcare clinics helps and coordinates care for patients as it may have potential benefits to reduce the communication gap between the two facilities (O'Malley \& Reschovsky, 2011; Spahr, Coddington, Edwards, \& McComb, 2018). Several studies looked at the usage of phone calls and short messaging services as reminders to patients, and it shows an increase in appointment attendance in general medicine (Jemere et al., 2019; Lee \& McCormick, 2003; Leong et al., 2006). In this study, the use of a mobile phone communication application is the communication medium of choice between clinic personnel and hospital personnel in sharing patient attendees' information which facilitates tracing of no-show for hospital appointments. In some studies, the usage of mobile phone applications for communication has facilitated timely responses among health teams in clinical settings (Ellanti, Moriarty, Coughlan, \& McCarthy, 2017; Kamel Boulos, Giustini, \& Wheeler, 2016). However, its usage as an information-sharing communication tool could be considered as individual-dependent, as it is the individual that perceives its usefulness in communication with other healthcare providers (De Benedictis et al., 2019). Another method of the patient tracing mechanism is utilising clinic healthcare personnel that go for home visits in their designated communities to help make physical tracing more feasible. Prompt tracing of patients on the same day also showed a reduction in missed appointments (Alamo et al., 2012).

Communications between leaders need to be properly established between the facilities. Leadership plays an important role in improving the implementation of the intervention and in the transition process into the new method from the old. Dialogues are important for leaders to exchange ideas with all sides; taking into account all opinions and concerns before making decisions (Al-Sawai, 2013; Sheikh, Ranson, \& Gilson, 2014). This would essentially facilitate the implementation and allay any concerns through an established discussion platform.

Any plans to scale up the referral mechanism should take into consideration the limitations mentioned in this evaluation. The use of technology, specifically seamless interoperable enterprise architecture between the facilities, may facilitate communication and access, consequently improving the safety, efficiency, and quality of care 
(Senitan et al., 2019; WHO).

Limitations. Findings for this study is limited to the views of HCP at the intervention clinics, intervention design team, state coordinators, while there were none from hospital specialists' and the patients' point of views. Hence, the results of this study cannot be generalized to all clinics outside of the intervention. In such case, to which the intervention would be upscaled, the views from these studies may be applicable. Therefore, this warrants a further study to explore and evaluate the EnPHC's referral system from the perspectives of the hospital specialists receiving the referral and patients who underwent the referral experience.

\section{Conclusion}

The EnPHC's referral mechanism created a platform for PHC clinics and hospitals to communicate and ensure care continuity for NCD patients. The availability of adequate information in the NCD care form helps the receiving physician to plan the treatment according to urgency. An established working communication platform between PHC clinics and hospitals helped build rapport in facilitating the monitoring of patients' compliance with hospital appointments, which may indirectly reduce the number of patients defaulting on their hospital appointments. It also allows the HCPs in PHC clinics to identify appointment defaulters and formulate treatment plans according to their needs.

\section{Acknowledgements}

The authors would like to express our gratitude to the Director-General of Health ef Malaysia for his permission to publish this paper. We would like to thank the Project Management Team for Enhanced Primary Healthcare, the State Directors of Johor and Selangor as well as the primary health care staff in both states for their contribution and participation in this study. We also would like to extend our gratitude to the Harvard T.H. Chan School of Public Health for their input on the development of study protocols.

\section{Authors' Contributions}

ZA, LLL, IAR \& SA were responsible for the concept, development, and supervision of the research and analyses of the data. ZA, LLL, IAR, SA, MZJ \& NHN constructed the draft manuscript. All authors contributed to the writing of the manuscript, reviewing and approving the final manuscript.

\section{Competing Interests Statement}

Zalilah Abdullah (ZA), Low Lee Lan (LLL), Iqbal Ab Rahim (IAR), Syafinaz Azam (SA), and Mohammad Zabri Johari (MZJ) are the research members and have received funding for the conduct of this study before publication. Nazrila Hairizan Nasir (NHN) was originally from the development team and joined the manuscript writing process. All other authors declare no conflict of interest in the writing of this paper.

\section{References}

Abdelwahid, H. A., Al-Shahrani, S. I., Elsaba, M. S., \& Elmorshedi, W. S. (2010). Patterns of referral in the Family Medicine Department in Southeastern Saudi Arabia. Saudi Med J, 31(8), 925-930.

Akbari, A., Mayhew, A., Al-Alawi, M. A., Grimshaw, J., Winkens, R., Glidewell, E., . . F Fraser, C. (2008). Interventions to improve outpatient referrals from primary care to secondary care. Cochrane Database Syst Rev, (4), Cd005471. https://doi.org/10.1002/14651858.CD005471.pub2

Al-Sawai, A. (2013). Leadership of healthcare professionals: where do we stand? Oman medical journal, 28(4), 285-287. https://doi.org/10.5001/omj.2013.79

Alamo, S. T., Wagner, G. J., Sunday, P., Wanyenze, R. K., Ouma, J., Kamya, M., . . Wabwire-Mangen, F. (2012). Electronic medical records and same day patient tracing improves clinic efficiency and adherence to appointments in a community based HIV/AIDS care program, in Uganda. AIDS Behav, 16(2), 368-374. https://doi.org/10.1007/s10461-011-9996-9

Ang, K. T., Ho, B. K., Mimi, O., Salmah, N., Salmiah, M. S., \& Noridah, M. S. (2014). Factors influencing the role of primary care providers as gatekeepers in the Malaysian public healthcare system. Malays Fam Physician, 9(3), 2-11.

Bodenheimer, T. (2008). Coordinating care--a perilous journey through the health care system. $N$ Engl J Med, 358(10), 1064-1071. https://doi.org/10.1056/NEJMhpr0706165

De Benedictis, A., Lettieri, E., Masella, C., Gastaldi, L., Macchini, G., Santu, C., \& Tartaglini, D. (2019). WhatsApp in hospital? An empirical investigation of individual and organizational determinants to use. PLoS One, 14(1), e0209873. https://doi.org/10.1371/journal.pone.0209873 
Ellanti, P., Moriarty, A., Coughlan, F., \& McCarthy, T. (2017). The Use of WhatsApp Smartphone Messaging Improves Communication Efficiency within an Orthopaedic Surgery Team. Cureus, 9(2), e1040. https://doi.org/10.7759/cureus. 1040

Forrest, C. B., Shadmi, E., Nutting, P. A., \& Starfield, B. (2007). Specialty referral completion among primary care patients: results from the ASPN Referral Study. Ann Fam Med, 5(4), 361-367. https://doi.org/10.1370/afm.703

Gandhi, T. K., Sittig, D. F., Franklin, M., Sussman, A. J., Fairchild, D. G., \& Bates, D. W. (2000). Communication breakdown in the outpatient referral process. $J$ Gen Intern Med, 15(9), 626-631. https://doi.org/10.1046/j.1525-1497.2000.91119.x

Gioia, D. A., Corley, K. G., \& Hamilton, A. L. (2012). Seeking Qualitative Rigor in Inductive Research: Notes on the Gioia Methodology. Organizational Research Methods, 16(1), 15-31. https://doi.org/10.1177/1094428112452151

Greenwood-Lee, J., Jewett, L., Woodhouse, L., \& Marshall, D. A. (2018). A categorisation of problems and solutions to improve patient referrals from primary to specialty care. BMC Health Services Research, 18(1), 986. https://doi.org/10.1186/s12913-018-3745-y

Jarallah, J. S. (1998). Referral from primary care to hospitals in saudi arabia: 1) quality of referral letters and feedback reports. Journal of family \& community medicine, 5(2), 15-22. Retrieved from https://pubmed.ncbi.nlm.nih.gov/23008585

Jemere, A. T., Yeneneh, Y. E., Tilahun, B., Fritz, F., Alemu, S., \& Kebede, M. (2019). Access to mobile phone and willingness to receive mHealth services among patients with diabetes in Northwest Ethiopia: a cross-sectional study. BMJ Open, 9(1), e021766. https://doi.org/10.1136/bmjopen-2018-021766

Jiwa, M., Walters, S., \& Mathers, N. (2004). Referral letters to colorectal surgeons: the impact of peer-mediated feedback. The British journal of general practice: the journal of the Royal College of General Practitioners, 54(499), 123-126. Retrieved from http://europepmc.org/abstract/MED/14965392

Kamel Boulos, M., Giustini, D., \& Wheeler, S. (2016). Instagram and WhatsApp in Health and Healthcare: An Overview. Future Internet, 8, 37. https://doi.org/10.3390/fi8030037

Lee, C. S., \& McCormick, P. A. (2003). Telephone Reminders to Reduce Non-Attendance Rate for Endoscopy. Journal of the Royal Society of Medicine, 96(11), 547-548. https://doi.org/10.1177/014107680309601108

Leong, K. C., Chen, W. S., Leong, K. W., Mastura, I., Mimi, O., Sheikh, M. A., . . Teng, C. L. (2006). The use of text messaging to improve attendance in primary care: a randomized controlled trial. Fam Pract, 23(6), 699-705. https://doi.org/10.1093/fampra/cml044

Merican, M. I., \& bin Yon, R. (2002). Health care reform and changes: the Malaysian experience. Asia Pac J Public Health, 14(1), 17-22. https://doi.org/10.1177/101053950201400105

Ministry of Health Malaysia. (2017). Enhance Primary Healthcare Lab (1st ed.). Putrajaya: Ministry of Health Malaysia.

O'Malley, A. S., \& Reschovsky, J. D. (2011). Referral and consultation communication between primary care and specialist physicians: finding common ground. Arch Intern Med, 171(1), 56-65. https://doi.org/10.1001/archinternmed.2010.480

Ramanayake, R. P. (2013). Structured printed referral letter (form letter); saves time and improves communication. $J$ Family Med Prim Care, 2(2), 145-148. https://doi.org/10.4103/2249-4863.117404

Senitan, M., Alhaiti, A. H., \& Gillespie, J. (2019). Improving integrated care for chronic non-communicable diseases: A focus on quality referral factors. International Journal of Healthcare Management, 12(2), 106-115. https://doi.org/10.1080/20479700.2018.1423663

Senitan, M., Alhaiti, A. H., \& Lenon, G. B. (2018). Factors contributing to effective referral systems for patients with non-communicable disease: evidence-based practice. International Journal of Diabetes in Developing Countries, 38(1), 115-123. https://doi.org/10.1007/s13410-017-0554-5

Shannon, D. (2012). Effective physician-to-physician communication: an essential ingredient for care coordination. Physician Exec, 38(1), 16-21.

Sheikh, K., Ranson, M. K., \& Gilson, L. (2014). Explorations on people centredness in health systems. Health policy and planning, 29 (Suppl 2), ii1-ii5. https://doi.org/10.1093/heapol/czu082 
Spahr, J., Coddington, J., Edwards, N., \& McComb, S. (2018). Implementing Comprehensive Primary Care Referral Tracking in a Patient-Centered Medical Home. Journal of Nursing Care Quality, 33(3), 255-262. https://doi.org/10.1097/NCQ.0000000000000283

van Dijk, C. E., de Jong, J. D., Verheij, R. A., Jansen, T., Korevaar, J. C., \& de Bakker, D. H. (2016). Compliance with referrals to medical specialist care: patient and general practice determinants: a cross-sectional study. BMC Family Practice, 17(1), 11. https://doi.org/10.1186/s12875-016-0401-7

Wåhlberg, H., Valle, P. C., Malm, S., \& Broderstad, A. R. (2015). Impact of referral templates on the quality of referrals from primary to secondary care: a cluster randomised trial. BMC Health Services Research, 15(1), 353. https://doi.org/10.1186/s12913-015-1017-7

WHO. (n.d.). Management of health facilities: Referral systems. Retrieved from http://www.who.int/management/facility/referral/en/

Wong, J., Tu, K., Bernatsky, S., Jaakkimainen, L., Thorne, J. C., Ahluwalia, V., . . Widdifield, J. (2019). Quality and continuity of information between primary care physicians and rheumatologists. BMC Rheumatology, 3(1), 23. https://doi.org/10.1186/s41927-019-0067-6

Woolf, S. H., Kuzel, A. J., Dovey, S. M., \& Phillips, R. L., Jr. (2004). A string of mistakes: the importance of cascade analysis in describing, counting, and preventing medical errors. Annals of family medicine, 2(4), 317-326. https://doi.org/10.1370/afm.126

\section{Copyrights}

Copyright for this article is retained by the author(s), with first publication rights granted to the journal.

This is an open-access article distributed under the terms and conditions of the Creative Commons Attribution license (http://creativecommons.org/licenses/by/4.0/). 